\title{
Medical Image Compression Using Hybrid Techniques of DWT, DCT and Huffman Coding
}

\author{
Thaneshwar Kumar ${ }^{1}$, Dr. Ramesh Kumar ${ }^{2}$ \\ M.Tech Scholar $\left(4^{\text {th }}\right.$ Sem $)$, BIT Durg ${ }^{1}$ \\ Prof., CSE Dept BIT Durg ${ }^{2}$
}

\begin{abstract}
Image compression plays a crucial role in medical imaging allowing efficient storage and transmissions by reducing the amount of data required to represent the digital image. The main goal is to achieve higher compression ratios and minimum degradation in quality. To decrease the storage space, the use of different compression techniques is justified by some medical imaging modalities generate the volume that of data which will be increasing. Different medical images like X-ray angiograms, magnetic resonance images, Ultrasound and computed Tomography are used in the medical image compression techniques. In medical applications it is required to conserve the diagnostic validity of the image requires the use of lossless compression methods, producing low compression factors. For medical data, lossless compression is preferred to the greater gains of lossy compression, in the interest of accuracy. . A set of experiment has been performed for the analysis of the proposed work on the several DICOM medical images and it has been observed that the DWT, DCT and Huffman coding has higher compression ratio than the hybrid model. The proposed method gives better quality of image that includes high PSNR and CR as well as low MSE. The proposed medical 'DICOM images compression scheme' is based on Hybrid DWT, DCT and Huffman coding techniques.
\end{abstract}

Keywords: DICOM images, DWT, DCT, Huffman coding, JPEG, PSNR, MSE, CR, Image compression, MATLAB.

\section{INTRODUCTION}

The objective of image compression is to reduce the images at various health care facilities is huge and redundancy of an image. Where loss of any Information is growing, and requirements of storage surpass the current not acceptable and data are critical then Lossless archival capacity. For example, the University of Compression method is applied. Compression of medical Washington Medical Center, a medium-sized hospital with image is based on lossless compression method. For about 400 beds, performs approximately 80000 studies per identification of disease and surgical planning medical year. At 30 Mbytes per study, the amount of digital images imaging is being used, and long-term storage is needed for generated is 2.4 Tera bytes of data per year or profiling patient's data. To avoid loss of critical medical approximately 10 Gbytes per day. To mitigate these information, lossless compressions of the medical images problems Image compression can be used. The image are indispensable. In the preceding few years, the compression techniques can be broadly classified into two generation of medical images in hospitals has been categories as Lossless and Lossy Compression. In Lossless increased considerably. In a typical hospital, large amount Compression, the original image can be reconstructed of medical data are generated every year. Compression has from the compressed image. Since they do not add noise to two catalogues. There are two techniques either lossy or an image, this technique is widely used in medical lossless techniques can be employed, it depends on the imaging. In Lossy compression technique, the system requirements. Complete data fidelity is ensured reconstructed image contains some degradation as after reconstruction by lossless compression, but in compared to the original one but it is nearly close to it. general compression ratios is limited between 2:1 to 3:1. This technique provides much higher compression ratios However, only ordinary reduction is provided in file size than the lossless scheme.

used by lossless techniques. Lossy compression techniques Lossy image compression:

are required to significantly affect storage costs. This is Lossy methods are especially suitable for natural images always taking into consideration that for the specific such as photographs in applications where minor clinical issue, loss must not be diagnostically significant. (sometimes imperceptible) loss of fidelity is acceptable to Medical images require large amounts of memory. For achieve a substantial reduction in bit rate. example, with 50-micron resolution and a 12-bit dynamic range is above 25 Mbytes amount is required by one image, and for each patient, there are four images. For medical imaging users, major issues are the consequent requirements of storage space. On the accuracy of clinical diagnosis It has become a crucial area for research to estimate the effect of image compression. The most usually used dimensions of image quality is peak signal noise ratio and mean square error are demonstrably inadequate for medical images. The generation of digital

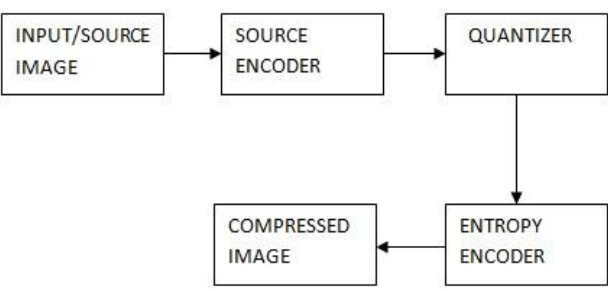

Fig.1: Lossy Image Compression 
Lossless Image Compression:

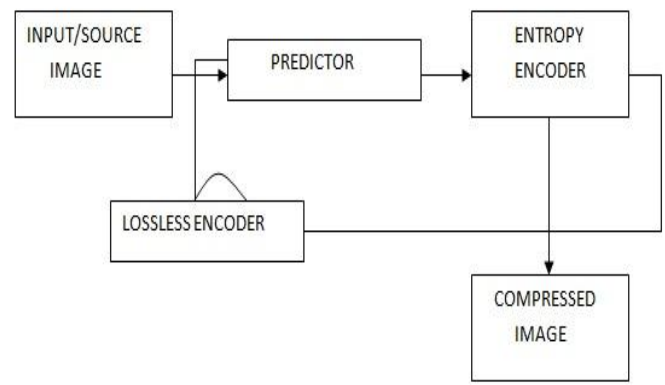

Fig. 2: Lossless Image Compression

Lossless compressions are preferred for archival purposes and often for medical imaging, clip art technical drawings etc.

\section{REVIEW OF LITERATURE}

Kitty Arora et al. in 2014 proposed A Comprehensive Review Of Image Compression Technique .The analysis of various compression techniques provides knowledge in the identifying the advantageous features and help in choosing correct method for compression. In the survey of different image compression techniques have been discussed from which researchers can get an idea for efficient techniques to be used. Shiby Angel K et al. in 2014 suggested the Medical Image Analysis and Processing Using a Dual Transform. An efficient method for compression of medical images is compared and studied with different metrics and algorithms. The experimental results determines how the compression ratio (CR), peak signal to noise ratio (PSNR) and SNR (signal to noise ratio) of different compression algorithms responds to dual transform algorithm. This dual transform method highly preserves quality of images with high compression ratio and bits per pixel. The quality of the image is also improved in terms of PSNR. K.N. Bharath et al. in 2013 proposed The Hybrid Compression Using DWT-DCT and Huffman Encoding Techniques for Biomedical Image and Video Applications. The approach of combining multiple transformations i.e. DWT-DCT, and Huffman coding has been successfully presented in this research work. The hybrid algorithm compensated the demerits of standalone DCT and DWT. Prof. Mukta Bhatele et al. in 2012 suggested Lossless Compression of Medical Images using Wavelet transform and Enhancing its Security. Hence modifying Huffman's technique and optimizing it, yields a more effective compression algorithm that increases the compression ratio on medical images. Hence effective compression technique results in a reduction in storage space, thereby improving the bandwidth and speed of transmission of medical images with no added complexity and resources. Prabhjot Kaur et al. in 2012 proposed Hybrid Huffman technique for medical image application. It is a new compression technique which is focus on lossless and lossy mechanism. This technique is suited for both lossy and lossless compressions. Here we are implement the hybrid Huffman and multithresholding method. We implement lossless technique so our PSNR and MSE will go better than the old algorithms and due to multithresholding we will get good level of compression. Arif Sameh Arif et al. in 2012 proposed Lossless Compression of Fluoroscopy Medical Images using Correlation and the Huffman Coding. A new framework for image compression based on the grouping the images based on the correlation has been proposed. The technique concentrates on the region of interest to code the difference between the groups of images using the combination of Run-length and Huffman coding. The method has achieved significant improvement in compression performance, and indirectly storage and transmission benefits. Qiusha Min et al. in 2010 presented a Hybrid Lossless compression scheme for efficient delivery of Medical image data over the internet. Unlike other 3D-based compression schemes of higher complexity and slower decompression speed, our proposed scheme can achieve high compression ratio with fast decompression time. It is applied to real volumetric medical image data and can be easily embedded into an online image viewer. Dimitrios A. Karras et al. in 2007 proposed Two novel image compression schemes for medical images based on the 2-D Discrete Wavelet Transform, the k-means based clustering of either the Discrete Wavelet Transform transform domain or the original image domain in terms of textural descriptors based criteria and the Bayesian restoration. Robina Asraf et al. in 2006 observed Diagnostically Lossless Compression-2 of Medical images. There many factors effect compression in proposed approaches they have chosen Huffman as their Lossless Compression technique Lempel Ziv coding may be another choice for lossless compression. The results can be improved if number of training vectors presented to NNVQ, and number of epochs to train NNVQ are increased. M. Nadir Kumaz et al in 2002 suggested compression of the MR and Ultrasound images by using wavelet transform. It was apparent that compressed biomedical images using the JPEG method at higher CRs contained a high amount of blocking noise. Although the WCM had also introduced some noise, this noise was not visually perceived until higher CRs .wavelet filters were searched to increase both the compression ratio and the reconstructed image quality. Liang Shen et al. in 1997 proposed A SegmentationBased Lossless Image Coding Method for HighResolution Medical Image Compression. Recent issues of journals in the field have published three review papers on medical image compression and one review on lossless image compression; none of these papers has provided new directions for lossless compression.

\section{PROBLEM IDENTIFICATION}

An efficient compression technique based on Discrete Wavelet Transform is proposed and developed. A set of test images (bmp format) are taken to justify the effectiveness of the algorithm A new image compression scheme based on discrete wavelet transform is proposed in this research which provides sufficient high compression ratios with no appreciable degradation of image quality. Discrete Wavelet Transformation is actually for timelimited data hence that maintains better image quality. So 
the Discrete Wavelet Transform is applied to an image and b. scaling filter (basically a low pass filter) : low the energy compaction performance of both Discrete frequency information is kept, high frequency information Cosine Transform and Discrete Wavelet Transform is is lost.

compared. It is observed that both transforms provide comparable energy compaction performance. An analysis and comparison of image compression using DCT and DWT. Since information loss implies some trade-off between error and bit rate, the measure of distortion (square error) is calculated. It is observed that different bands provide low pass information, and horizontal, vertical and diagonal edges. It is also observed that both transforms provide comparable energy compaction performance. In the case of medical images there is a great need to have no deterioration in image quality. The result proves that with using a Dual Transform there is no losses in quality of images, it can be used in the application of medical purposes. The implementation of hybrid Huffman technique is, so that PSNR, high CR and MSE will go better than the old algorithms and due to multithresholding get good level of compression. Huffman encoding is a lossless data compression technique. At the most optimal compression the original and uncompressed from wavelet coefficient is almost the very same. Future scope depends on developing a trained system which can automatically detect type of medical image and determine which suitable wavelet will produce the best compression on it. With the combination of both DCT-Discrete Wavelet Transform Techniques, an image having larger dimension can be compressed by Discrete Wavelet Transform using multiresolution analysis and once the image gets small dimension, it is compressed easily by DCT-technique. So, we can obtain better PSNR value than individuals.

\section{METHODOLOGY}

Discrete Wavelet Transform (DWT)

Discrete Wavelet Transform is having data reduction capability which has become a standard tool in image compression applications. As in a Discrete Cosine Transform based compression system, the entire image is transformed and compressed as a single data object rather than block by block in Discrete Wavelet Transform. Wavelet analysis can be used to divide the information of an image into approximation and detailed sub signal. Compared with Fourier transform, the wavelet transform is space (time) and frequency of local transform, so it can effectively extract information from signal. The Discrete Wavelet Transform separates an image into a lower resolution approximation image (LL) as well as horizontal (HL), vertical (LH) and diagonal (HH) detail components. Wavelets are having an average value equal to zero and they are defined over all. The wavelet called mother wavelet is the basis function which is obtained from a single prototype wavelet. The basis functions include wavelet function and scaling function. The image is first divided into blocks and each block is then passed through the two filters:

a. wavelet filter (basically a high pass filter) : high frequency information is kept, low frequency information is lost.

After doing the first level of decomposition, four sub images are formed namely LL, LH, HL, and $\mathrm{HH}$ coefficients. A nonreversible filter is used for this transformation. So signal is effectively decomposed into two parts, a detailed part (high frequency) and approximation part (low frequency).

\section{Discrete Cosine Transform (DCT)}

Discrete Cosine Transform is a lossy Compression technique which is widely used in area of image and audio compression. Example: JPEG Images. Discrete Cosine Transforms are used to convert data into the summation of series of cosine waves is oscillating at different frequencies. These are very similar to Fourier Transforms, but Discrete Cosine Transform involves use of cosine functions and real coefficients, Fourier Transforms use both sine and cosine and cosine functions are much more efficient as fewer functions are needed to approximate a signal. Both Fourier and Discrete Cosine Transform convert data from a spatial domain into a frequency domain and their respective functions converting thing back. In the Discrete Cosine Transform, the images are separated into different parts of varying importance. Discrete Cosine Transform expresses a sequence of finitely several data points oscillating at different frequencies in terms of sum of cosine functions. Similarly as the discrete Fourier transform (DFT), a Discrete Cosine Transform is also a Fourier-related transform, but using only real numbers. The inversion of Discrete Cosine Transform can be accomplished hence the Discrete Cosine Transform is a unitary transform . The Discrete Cosine Transform helps to separate the image into spectral subbands of differing importance with respect to the visual quality of images. The Discrete Cosine Transform is similar to the domain. In a Discrete Cosine Transform, a sequence of finitely many data points oscillating at different frequencies are expressed in terms of a sum of cosine functions. To spectral for the numerical solution of partial differential equations from lossy compression of images (e.g. JPEG) where small high-frequency components can be discarded, Discrete Cosine Transforms are important to numerous applications in science and engineering. Rather than the sine functions use of cosine is critical in these applications: for compression, it turns out that cosine functions are much more efficient (fewer are needed to approximate a typical signal as described), whereas for differential equations a particular choice of boundary conditions are expressed by the cosines.

\section{Huffman Coding}

Proposed by DR. David A. Huffman in 1952. A method for the construction for minimum redundancy code. Huffman code is technique for compressing data. Huffman made significant contributions in several areas. Mostly information theory and coding signal design procedures 
for asynchronous logical circuits and design for radar and Finally dictionary 2 is obtained. Lossless compression communication. Huffman coding is a form of statistical coding which attempt to reduce the amounts of bits required representing the string of symbols to vary in length. Shorter codes are assigned to the most frequently used symbols \& longer codes to the symbol which appear less frequently in the string. Code word length is no longer fixed like ASCII. In the early 1980s, personal computers had hard disks that were no larger than 10MB; today, the puniest of disks are still measured in hundreds of gigabytes. Even though hard drives are getting bigger, the files we want to store (funny pictures of cats, videos, music and so on) seem to keep pace with that growth which makes even today's gargantuan disk seem too small to hold everything. One technique to use our storage more optimally is to compress the files. By taking advantage of redundancy or patterns, we may be able to "abbreviate" the contents in such a way to take up less space yet maintain the ability to reconstruct a full version of the original when needed. Such compression could be useful when trying to cram more things on a disk or to shorten the time needed to copy/send a file over a network.

\section{Hybrid (DWT, DCT \& Huffman Coding) Techniques}

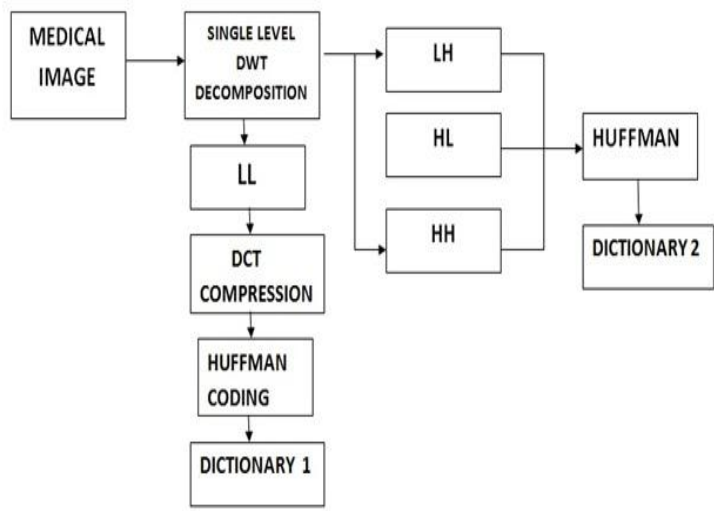

schemes are extremely suitable for text compression in which they provides high compression ratio without any data loss but in image compression ratio we use lossy compression scheme for getting high compression ratio. By using lossy compression scheme we also lose some data and in medical image processing there should be minimum loss of data which do not affect the end results. Wavelet compression provides high data compression ratio and minimized the Mean square error and to maximize the peak signal to noise ratio. So Hybrid Compression technique is best for medical image compression.

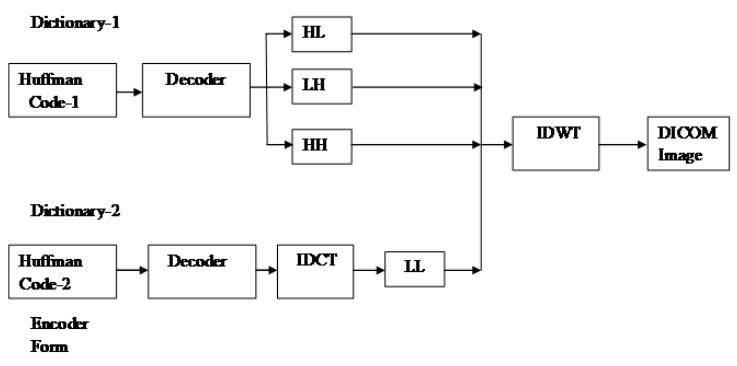

Fig. 4: Basic Image Decompression Block Diagram Of Hybrid Technique.

This process is just reverse of the compression process where all the three major technique have been hybridized to decompress the image. Huffman Encoded Dictionary 1 for High Frequency component as Dictionary 2 for the low level frequency component. Huffman Decoding is processed on both the dictionaries to get the high level frequency component and Discrete Cosine Transform is used to compress Image. Applying IDCT in the DCT compressed image to get low level LL frequency component. Apply IDWT all the four frequency component to get the Decompressed image

\section{RESULT AND DISCUSSION}

Fig. 3: Basic Image Compression Block Diagram of Hybrid Technique.

Hybrid Discrete Wavelet Transform and Discrete Cosine Transform techniques are the algorithm developed for medical image compression. To calculate the Compression Ratio (CR), Peak Signal to Noise Ratio (PSNR) and Mean Square Error (MSE) is the objective of this Hybrid (DWT,DCT and Huffman coding) scheme. The goal is to achieve higher compression ratio while preserving the quality of reconstructed image. The presented hybrid DWT, DCT algorithm for medical image compression is to exploit the properties of both the DWT and DCT (as illustrated in fig. 3) .take the different medical image size $175 \mathrm{~kb}$. First step involves single level decomposition of Discrete Wavelet Transform and different four level of bands are (LL, LH, HL \& HH) obtained after applying first step on medical images. Second step involves Discrete Cosine Transform compression on LL band. Next step involves the Huffman coding on this LL band and dictionary 1 is obtained. Now the Huffman coding is applying on remaining three bands (LH, HL \& HH).

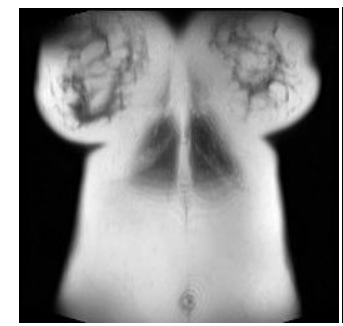

Fig. 5: Chest

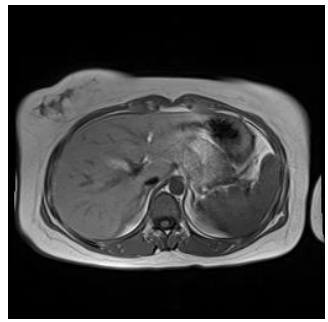

Fig.7: Liver

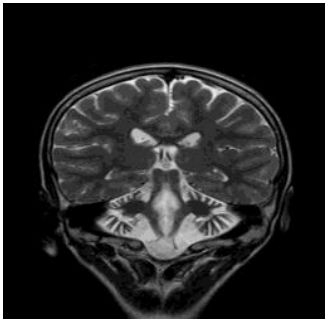

Fig. 6: Head

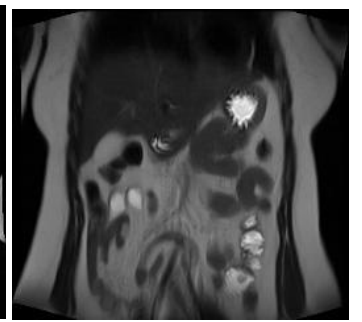

Fig. 8: Abdomen 


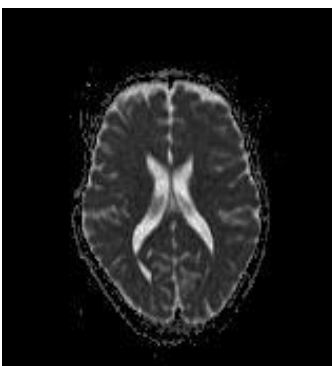

Fig. 9: Brain

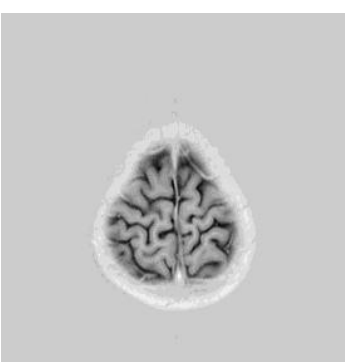

Fig. 10: Brain
The experiments are carried out to demonstrate the effect of the proposed schemes. In experiments, we adopt medical DICOM images with size $192 \times 256$ as the original images and the $175 \times 175$ as the medical DICOM image. The technique is implemented in MATLAB 2012 environment. The input database images taken for the experimental purpose are Brain, Chest, Head, Lungs, Spinal Cord, Skull; all images are in jpg format (jpg image file). Figure below shows the DICOM image files for the five input cover images. Here fig. 5 is chest, 6 is Head, 7 is Liver, 8 is Abdomen, 9 is Brain and 10 is a part of brain, shows how various DICOM medical images are taken as an input by our compression technique.

\section{Comparative Analysis of DCT, DWT and Hybrid Technique :}

Figure 11 shows graph of comparative analysis of DWT, DCT, Hybrid technique with respect to MSE. And it is concluded that DWT shows less error compare to other, hence its performance is better to other technique.

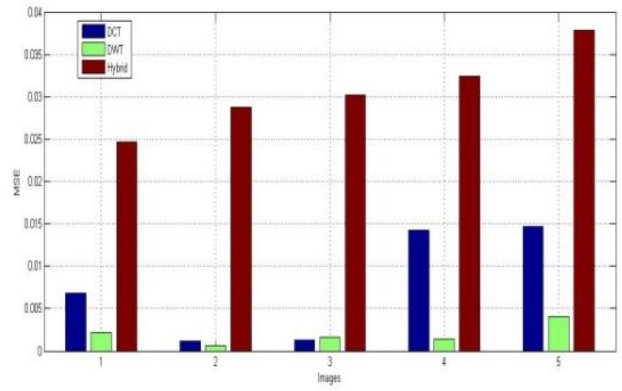

Fig. 11 Graph for MSE

Figure 12 shows the graphical representation of values that is obtained by DWT, DCT, hybrid compression technique with respect to PSNR. Here by graphs its clear that DWT compression shows higher values and gives better performance then other technique.

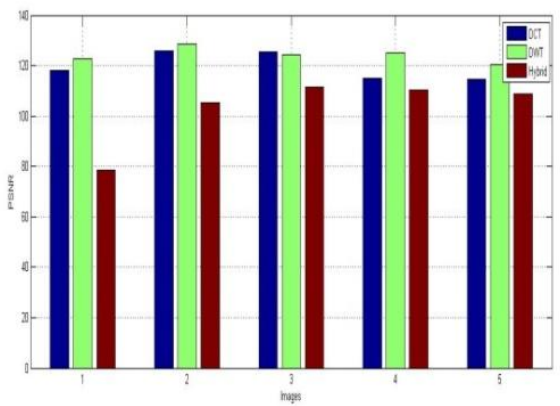

Fig. 12: Graph for PSNR

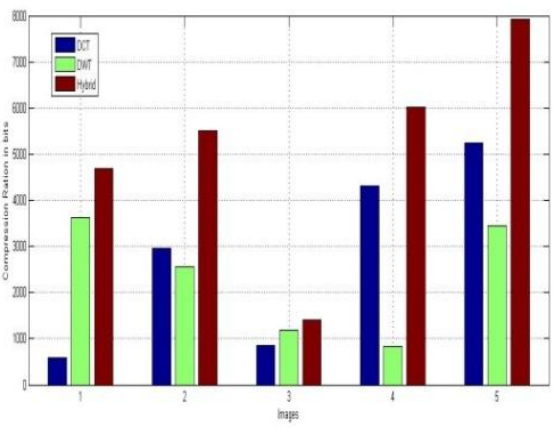

Fig. 13: Graph for CR

Figure 13 shows graphical comparison of DWT, DCT, and Hybrid compression technique with respect to CR. And it's concluded that Hybrid compression shows better performance than other compression technique.

\section{MSE: Mean-Square error.}

The MSE represents the cumulative squared error between the compressed and the original image, whereas PSNR represents a measure of the peak error. The lower the value of MSE, the lower the error. To compute the PSNR, the block first calculates the mean squared error using the following equation:

$$
\mathrm{MSE}=\sum_{i=1}^{x} \sum_{j=1}^{y} \frac{\left(\left|A_{i j}-B_{i j}\right|\right)^{2}}{x * y}
$$

$\mathrm{X}$ : width of image.

Y: height.

$\mathrm{X}^{*} \mathrm{y}$ : number of pixels (or quantities).

Table: 1 Comparison of DCT, DWT \& Hybrid based approach in terms of MSE

\begin{tabular}{|l|l|l|ll|}
\hline S.NO & DICOM ME DICAL IMAGES & DCT & DWT & HYBRID \\
1 & Image set 1 & 0.006817 & 0.002195 & 0.0246 \\
\hline 2 & Image set 2 & 0.00113 & 0.000615 & 0.1239 \\
\hline 3 & Image set 3 & 0.0012333 & 0.001651 & 0.030142 \\
\hline 4 & Image set 4 & 0.014187 & 0.001332 & 0.041599 \\
5 & Image set 5 & 0.014673 & 0.004019 & 0.056473 \\
\hline
\end{tabular}

In table 1 MSE comparison of prepared method along with Discrete Cosine Transform, DWT and it is found not Discrete Wavelet Transform shows better performance as compared to other algorithm.

\section{PSNR (Peak Signal to Noise Ratio)}

The PSNR block computes the peak signal to noise ratio, in decibels between two images. This ratio is often used as a quality measurement between the original and a compressed image. The higher the PSNR, the better the quality of the compressed or reconstructed image. PSNR is generally used to analyze quality of image, sound and video files in $\mathrm{dB}$ (decibels). 


$$
\operatorname{PSNR}(\mathrm{dB})=10 * \log \left(\frac{255^{2}}{M S E}\right)
$$

Table: 2 Comparison of DCT, DWT \& Hybrid based approach in terms of PSNR

\begin{tabular}{|l|l|l|ll|}
\hline S.NO & DICOM ME DICAL IMAGES & DCT & DWT & HYBRID \\
1 & Image set 1 & 0.006817 & $\mathbf{0 . 0 0 2 1 9 5}$ & 0.0246 \\
\hline 2 & Image set 2 & 0.00113 & 0.000615 & 0.1239 \\
\hline 3 & Image set 3 & 0.0012333 & 0.001651 & 0.030142 \\
\hline 4 & Image set 4 & 0.014187 & 0.001332 & 0.041599 \\
5 & Image set 5 & 0.014673 & 0.004019 & 0.056473 \\
\hline
\end{tabular}

In table 2 PSNR comparison of proposed method along with Discrete Cosine Transform, DWT and it is found that Discrete Wavelet Transform shows better performance as compared to other algorithm.

Table: 3 Comparison of DCT, DWT \& Hybrid based approach in terms of CR.

\begin{tabular}{|l|l|l|ll|}
\hline S.NO & DICOM ME DICAL IMAGES & DCT & DWT & HYBRID \\
1 & Image set 1 & 0.006817 & 0.002195 & 0.0246 \\
\hline 2 & Image set 2 & 0.00113 & 0.000615 & 0.1239 \\
\hline 3 & Image set 3 & 0.0012333 & 0.001651 & 0.030142 \\
\hline 4 & Image set 4 & 0.014187 & 0.001332 & 0.041599 \\
5 & Image set 5 & 0.014673 & 0.004019 & 0.056473 \\
\hline
\end{tabular}

In table 3 CR Comparison of proposed method along with DCT, DWT and it is found that Hybrid shows better performance as compared to other algorithm.

CR (Compression Ratio)

$\mathrm{CR}=\frac{\text { Size of Original Image }}{\text { Size of Compressed Image }}$

Note : Image set1,image set2, up to image set5 has been prepared by taking 10 images of various body part images and the MSE,PSNR and CR are the average values the images present in each set.

\section{CONCLUSION}

In this paper we have proposed a medical DICOM images compression scheme based on Hybrid DWT, DCT and Huffman coding techniques. The algorithm based on Discrete Cosine Transform offers a robust method of medical DICOM images with minimum distortion. The Discrete Wavelet Transform have advantage to decomposition of images which is necessary criteria for it .The Huffman encoding reduces the average codeword length by assigning shorter codeword's to highly frequent symbols and longer codeword's to rarely occurring symbols. Thus these desirable properties have been utilized to create a new robust compression technique. In the proposed method a new compression technique which is focus on lossless mechanism. This technique is suited for lossless compressions. Result show that it gives better quality images \& high CR, high PSNR and low MSE are also improved. This hybrid compression technique is tested against different medical images using different values of compression factors (i.e. DWT \& DCT quantization factor). Different types of wavelet are also used to improve the imperceptibility. The extracted procedure reverses the embedding operation with the reference of original and compressed image. The technique is tested against 10 images of 5 set and total 50 images have been computed for the result.

\section{FUTURE SCOPE}

A new proposed hybrid scheme for medical image compression is revolution of DWT, DCT and Huffman coding algorithm. This technique is tested along with different medical images (DICOM) with different values of quantization factor. As the value of quantization factor gets increases, the compression ratio increases and the quality measurement (PSNR) decreases. The quality of an image after compression is the main criteria that all the compression techniques should hold. To conclude, all the compression techniques are useful for real-time medical image transmission or storage process. Every technique is different and gives suitable results for the each technique. Now a day's many compression techniques are evolving hence the selection of high PSNR value will lead to maintain the quality of an image and achievement is in compression process. This concludes that after applying lossy technique it is better to use lossless to enhance compression at the same PSNR. Hence in future a new compression method gives very good result and it leaves good probability for further expansion. This work can be expanded by applying it for the videos to get better PSNR at high CR.

\section{REFERENCE}

[1] Kitty Arora et al., "A Comprehensive Review of Image Compression Techniques" IJCSIT, vol.5 (2), pp.1169-117 2, 2014.

[2] H.B. Kekre et al., "Performance Comparison of Hartley Transform with Hartley Wavelet and Hybrid Hartley Wavelet Transforms for Image Data Compression" IJCT,vol.12,no.6, ISSN: 2277-3061,Feb.2014.

[3] Deepak Kumar Jain et al., "Image Compression Using Discrete Cosine Transform and Adaptive Huffman Coding", IJETTCS, vol.3, ISNN: 2278-6856, Feb.2014.

[4] Shiby Angel K, "Medical Image Analysis and Processing A Dual transform”,IJRET,vol.3, pp.325-329, 2014.

[5] Sandhya Sharma et al., "Image Compression Using Hybrid of DWT, DCT and Huffman Coding", IJSETT, vol.5(1), pp.19-23, 2013.

[6] Jayavrinda Vrindavanam et al., "Huffman Coding and Position Based Coding scheme for Image Compression: An Experimental Analysis", IJAIS, ISSN: 2249-0868, 2013.

[7] K.N. Bharat et al., "Hybrid Compression using DWT-DCT \& Huffman Encoding Techniques for Biomedical Image and Video Applications" IJCSMC, vol.2, pp.255-261, May.2013.

[8] Manisha Singh et al., "Image Compression with Hybrid Method: An Overview", IJEETC, vol.2, no.3, ISSN: 2319-2518, 2013.

[9] Pallavi M. Sune et al., "Image Compression Technique Based on Wavelet \& Huffman coding", IJARCSSE, vol.3, ISSN: 2277-128X, April.2013.

[10] Prof. Mukta Bhatele et al.,"Lossless Compression of Medical Images Using Wavelet Transform \& Enhancing It's Security" ,IJSAA.vol.2, ,ISSN :2277-2677, Jan.2012.

[11] Smitha Joyce Pinto et al., "Prformance Analysis Of Medical Image Compression Techniques", IEEE, 2012.

[12] Harjeetpal Sing et al., "Hybrid Image Compression uses DWT, DCT \& Huffman Encoding Techniques", IJETAE, vol.2, ISSN: 2250-2459, Oct.2012.

[13] Prabhjot Kaur et al.,"Hybrid Huffman Technique for Medical Image Application", IJARECE, vol.1, ISSN:2278-909X, Oct.2012.

[14] Alessandro Polpetta et al.,"Design \& Performance of Huffman Sequences in Medical Ultrasound Coded Excitation", vol. 59 ,no.4, IEEE, Apr.2012.

[15] Arif Sameh et al.,"Lossless Compression of Fluoroscopy Medical Images Using Correlation \& The Combination of Run- Length \& Huffman Coding”, IEEE, Dec.2012. 
[16] Geetika et al., "Novel Image Compression Technique with Improved Wavelet Method", IJRTE, vol.1, ISSN: 2277-3878, June.2012.

[17] Rashmi S. Nair et al., "Advanced Computing \& Communication Technologies for HPC Applications”, ACCTHPCA, ISSN: 09758887, Jun.2012.

[18] Aree Ali Mohammed et al., "Hybrid Transform Coding Scheme for Medical Image Application”,IEEE, 2011.

[19] Suchitra Shrestha et al.,"Hybrid DWT- DCT Algorithm for Biomedical Image \& Video compression Applications", ISSPA,2010.

[20] Qiusha Min et al., "A Hybrid Lossless Compression Scheme for Efficient Delivery of Medical Image Data over the Internet",ICCMS, 2010.

[21] S. Bhavani et al.,"A Survey on Coding Algorithms in Medical Image Compression”,IJCSE,vol.2,no.5,ISSN: 0975-3397, 2010.

[22] S.M. Ramesh et al.,"Medical Image using Wavelet Decomposion for Prediction Method", IJCSIS, vol.7, no.1,ISSN : 1947-5500,2010.

[23] M. Tamilarasi et al., "Contourlet Based Medical Image Compression using Improved EZW”, IEEE, 2009.

[24] Jayanta Kumar Debnath et al., "A Modified Vector Quantization Based Image Compression Technique using Wavelet Transform", IEEE, 2008.

[25] Dimitrios A. Karras, "Improved Video Compression Schemes of Medical Image Sequences Based on The Discrete Wavelet Transformation of Principal Textural Regions \& Intelligent Restoration Techniques", IEEE, 2007.

[26] V Naga Prudhvi Raj et al., "A Novel Approach to Medical Image Compression Using Sequential 3D- DCT”, IEEE, 2007.

[27] Chuo-Ling Chang et al., "Direction-Adaptive Discrete Wavelet Transform for Image Compression”, IEEE, May.2007.

[28] Robina Ashraf et al., "Absolutely Lossless Compression of Medical Images", IEEE, Sep.2005.

[29] Robina Ashraf et al., "Diagnostically Lossless Compression 2D of Medical Images" IEEE, Apr.2006.

[30] M. Sabarirnalai Manikandan et al., "Wavelet-Threshold based ECG Compression Smooth Retrieved Quality for Telecardiology", IEEE, 2006.

[31] Xiaofeng Li et al., "A Medical Image Compression Scheme based on Low Order Linear Predictor and Most- Likely Magnitude Huffman Code", IEEE, June.2006.

[32] Xiaofeng $\mathrm{Li}$ et al., "An efficient medical image compression scheme", IEEE, Sep.2005.

[33] Yen-Yu Chen et al., "Embedded Medical Image Compression using Discrete Cosine Transform based Subband Decomposition \& Modified SPIHT Data Organization", IEEE, 2004

[34] M. Nadir Kurnaz et al., "Compression of The MR \& Ultrasound Images by Using Wavelet Transform", IEEE, pp.1021-1022, Oct.2002.

[35] Sonja Grgic et al.,"Performance Analysis of Image Compression Using Wavelets”, IEEE, vol.48 ,no.3 , pp.682-695, Jun.2001.

[36] Markus H. Gross et al., "Compression Methods for Visualization", ETH, no.23, pp.1-20, 1998.

[37] Liang Shen et al., "A Segmentation- Based Lossless Image Coding Method for High- Resolution Medical Image Compression", IEEE, pp.301-307, Jun.1997. 